\title{
On lower bounds of exponential frames*
}

\author{
Alexander M. Lindner
}

\begin{abstract}
Lower frame bounds for sequences of exponentials are obtained in a special version of Avdonin's theorem on "1/4 in the mean" (1974) and in a theorem of Duffin and Schaeffer (1952).
\end{abstract}

Keywords: lower bound, exponential frame, sine-type-function, irregular sampling

Math Subject Classification: 42C15, 30A10, 94A12

\section{Introduction}

The notion of frame has been introduced by Duffin AND Schaeffer [3]. A sequence $\left(\varphi_{n}\right)_{n \in \mathbb{Z}}$ in a Hilbert space $\left(H,(\cdot, \cdot)_{H}\right)$ is a frame for $H$, if there exist positive constants $A, B$ such that for all $f$ in $H$ :

$$
A\|f\|_{H}^{2} \leq \sum_{n \in \mathbb{Z}}\left|\left(f, \varphi_{n}\right)_{H}\right|^{2} \leq B\|f\|_{H}^{2} .
$$

More specifically, $\left(\varphi_{n}\right)_{n \in \mathbb{Z}}$ is called an $(A, B)$-frame. The constants $A$ and $B$ are called lower and upper frame bounds, respectively. A frame is exact if it is no longer a frame after any of its elements is removed.

DUfFIN AND SCHAEFFER [3] have given a sufficient condition for a sequence of exponentials $\left(e^{i \lambda_{n} \bullet}\right)_{n \in \mathbb{Z}}$ to be a frame for $L^{2}(-\gamma, \gamma), \gamma>0$. Avdonin [1] has given a sufficient condition for a sequence of exponentials $\left(e^{i \lambda_{n} \bullet}\right)_{n \in \mathbb{Z}}$ to be an exact frame for $L^{2}(-\pi, \pi)$. In both papers, only the mere existence of a lower bound is proved.

D. KöLzow [6] asked for explicit lower frame bounds, in terms of the data by which the sequence $\left(\lambda_{n}\right)_{n \in \mathbb{Z}}$ is restricted. In this paper we shall obtain a lower bound in a special version of Avdonin's theorem on " $1 / 4$ in the mean" (Theorem 1). The result will be used to obtain a lower bound in a theorem of Duffin and Schaeffer (Theorem 2). Finally, an application to irregular sampling is pointed out.

${ }^{*}$ The results of this paper are part of the author's forthcoming doctoral thesis and were presented, in a preliminary form, at the 1997 International Workshop on Sampling Theory and Applications, June 16 - 19, 1997, Aveiro, Portugal. 


\section{Preliminary remarks and main results}

$P W_{\sigma}^{2}$ denotes the Paley-Wiener space of entire functions of exponential type at most $\sigma$, whose restriction to $\mathbb{R}$ belongs to $L^{2}(\mathbb{R})$. For a sequence $\left(\lambda_{n}\right)_{n \in \mathbb{Z}}$ of real numbers the classical Paley-Wiener theorem yields that $\left(e^{i \lambda_{n} \bullet}\right)_{n \in \mathbb{Z}}$ is an $(A, B)$ frame for $L^{2}(-\sigma, \sigma)$ if and only if

$$
A\|F\|_{P W_{\sigma}^{2}}^{2} \leq 2 \pi \sum_{n \in \mathbb{Z}}\left|F\left(\lambda_{n}\right)\right|^{2} \leq B\|F\|_{P W_{\sigma}^{2}}^{2} \quad \forall F \in P W_{\sigma}^{2} .
$$

A sequence $\left(\lambda_{n}\right)_{n \in \mathbb{Z}}$ of complex numbers is called separated by $\delta>0$, if

$$
\left|\lambda_{n}-\lambda_{m}\right| \geq \delta \quad \forall m, n \in \mathbb{Z}: m \neq n \text {. }
$$

The following lemma asserts an upper bound for separated sequences with bounded imaginary parts. The proof follows the same argument as in the proof of Lemma 2 in KATSNEL'SOn [4], using $\operatorname{card}\left\{n \in \mathbb{Z}:\left|z-\lambda_{n}\right| \leq 1\right\} \leq(1+2 / \delta)^{2} \forall z \in \mathbb{C}$.

Lemma 1. Let $\left(\lambda_{n}\right)_{n \in \mathbb{Z}}$ be a sequence of complex numbers, separated by $\delta>0$, with imaginary parts bounded by $\tau<\infty$. Then,

$$
\sum_{n \in \mathbb{Z}}\left|F\left(\lambda_{n}\right)\right|^{2} \leq \frac{2}{\pi}\left(\frac{2}{\delta}+1\right)^{2} \cdot \frac{e^{2 \sigma(\tau+1)}-1}{2 \sigma}\|F\|_{P W_{\sigma}^{2}}^{2} \quad \forall F \in P W_{\sigma}^{2}
$$

for any $\sigma>0$.

The main results of this paper are the following:

Theorem 1 (A lower bound in the Theorem of Avdonin). Let $\left(\delta_{k}\right)_{k \in \mathbb{Z}}$ be a sequence of real numbers, bounded by $L<\infty$. Suppose $\left(k+\delta_{k}\right)_{k \in \mathbb{Z}}$ is separated by $\delta>0$, and there are $d \in[0,1 / 4)$ and a natural number $N$, such that

$$
\left|\sum_{k=j N+1}^{(j+1) N} \delta_{k}\right| \leq N \cdot d \quad \forall j \in \mathbb{Z}
$$

Then, $\left(e^{i\left(k+\delta_{k}\right) \bullet}\right)_{k \in \mathbb{Z}}$ is an exact frame for $L^{2}(-\pi, \pi)$, and the following constant is a lower frame bound:

$$
A_{A v}(L, \delta, d, N):=e^{-20 \pi^{2}(2 \tilde{L})^{2 \tilde{N}} / \tilde{N}^{2}} \cdot\left(\frac{\tilde{\delta}}{9 \tilde{L}}\right)^{240(2 \tilde{L})^{\tilde{N}}}
$$

where

$$
\tilde{N}:=N \cdot\left\lceil\frac{1}{N} \cdot \frac{2(4 L+2)^{2}}{1 / 4-d}\right\rceil, \quad \tilde{L}:=\frac{3}{2}+2(3 L+1), \quad \tilde{\delta}:=\frac{1}{2}\left(\frac{1}{4}-d\right) \delta .
$$

( $\lceil x\rceil$ denotes the smallest integer greater than or equal to $x$.)

Theorem 2 (A lower bound in the Theorem of Duffin and Schaeffer). Suppose $\left(\lambda_{n}\right)_{n \in \mathbb{Z}}$ is a sequence of real numbers, separated by $\delta>0$. Let $\sigma>0, L \geq$ 
$0,0<\gamma<\pi \sigma$ and suppose $\left(\lambda_{n}-n / \sigma\right)_{n \in \mathbb{Z}}$ is bounded by L. Then, $\left(e^{i \lambda_{n} \bullet}\right)_{n \in \mathbb{Z}}$ is a frame for $L^{2}(-\gamma, \gamma)$, and the following constant is a lower frame bound:

$A_{D S}(L, \delta, \sigma, \gamma):=\frac{\gamma}{\pi} \cdot e^{-5 \pi^{2} \frac{(12 M+1)^{4 M(M+1)}}{M^{2}(M+1)^{2}}} \cdot\left(\frac{\delta \gamma / \pi}{308 M}\right)^{240(12 M+1)^{2 M(M+1)}}$,

where

$$
M:=\max \left\{13,\left\lceil\frac{3+2 \sigma L}{\pi \sigma / \gamma-1}\right\rceil\right\} .
$$

While the proofs of Avdonin [1, Theorem 2] and of Duffin And Schaeffer [3, Theorem I] only assured the existence of lower bounds, Theorems 1 and 2 give explicit lower bounds. The proof of Theorem 1 follows by explicating and supplementing that of Avdonin. Theorem 2 will follow from Proposition 1, by explicating a construction of SEIP [9, Theorem 2.3]. We remark that Avdonin's theorem was proved for more general sets of zeros of sine-type-functions, more general partitions and complex sequences $\left(\delta_{k}\right)_{k \in \mathbb{Z}}$. The proof of Theorem 1 works for this general situation as well, if suitable conditions on the sine-type-function and partition are posed.

\section{Proof of the theorems}

For the proof of Theorem 1, we will need the notion of sine-type-function.

Definition. An entire function $f$ of exponential type is called $\sigma$-sine-type-function $(\sigma>0)$, if the zeros of $f$ are simple and separated and there are $C_{1}, C_{2}, \tau>0$ such that

$$
C_{1} \cdot e^{\sigma|y|} \leq|f(x+i y)| \leq C_{2} \cdot e^{\sigma|y|} \quad \forall x, y \in \mathbb{R}:|y| \geq \tau
$$

Remark. The Theorem of Avdonin rests on the following result of Levin, proved indirectly: For any sine-type-function $f$ with zeros $\left(\lambda_{n}\right)_{n \in \mathbb{Z}}$, there are constants $C_{3}$, $C_{4}>0$ such that

$$
C_{3} \leq\left|f^{\prime}\left(\lambda_{n}\right)\right| \leq C_{4} \quad \forall n \in \mathbb{Z}
$$

(cf. Levin [7], [8, p. 164], Young [10, p. 173]). The proof does not yield any estimates for $C_{3}$.

The following lemma plays the central role in the proof of Theorem 1; it supplements Lemmas 1, 2 in AVDONIN [1]: the growth of the constructed sine-typefunction is determined and estimates for the derivatives at the zeros are found. (Let $1 / 2 \mathbb{Z}:=\{n / 2: n \in \mathbb{Z}\}$.)

Lemma 2. Let the assumptions of Theorem 1 be satisfied. Suppose furthermore that $\left(k+\delta_{k}\right)_{k \in \mathbb{Z}}$ is increasing and that

$$
N>2 L \cdot \frac{3 / 4+d}{1 / 4-d}
$$


Define

$$
\begin{gathered}
L^{\prime}:=3 / 2+2 L, \quad \delta^{\prime}:=(1 / 4-d) \delta \\
D_{\varepsilon}:=\left(\left(1+\frac{L^{\prime}}{\varepsilon}\right)\left(1+\frac{8 L^{\prime}}{\delta^{\prime}}\right)\right)^{4 \sqrt{4 L^{\prime} N^{2}+2\left(2 L^{\prime}\right)^{2 N}}+6 N} \cdot e^{\left(2 L^{\prime}+\left(2 L^{\prime}\right)^{2 N} / N^{2}\right) \pi^{2}} \forall \varepsilon>0, \\
C_{1}:=\left(\frac{1-e^{-2 \pi}}{2}\right)^{2} D_{1}^{-1}, C_{2}:=D_{1}, \\
C_{3}:=\frac{\pi^{2} \delta^{\prime} e^{-\pi^{2} / 4}}{32} D_{\delta^{\prime} / 8}^{-1}, C_{4}:=\frac{2 \pi^{2} e^{\pi^{2} / 4}}{\delta^{\prime}} D_{\delta^{\prime} / 8} .
\end{gathered}
$$

Then, there is a sequence $\left(\delta_{k+1 / 2}\right)_{k \in \mathbb{Z}}$ of real numbers and a $2 \pi$-sine-type-function $\Psi$, real on the real axis, with zeros $\left(k+\delta_{k}\right)_{k \in 1 / 2 \mathbb{Z}}$, such that $\left(\delta_{k}\right)_{k \in 1 / 2 \mathbb{Z}}$ is bounded by $L^{\prime},\left(k+\delta_{k}\right)_{k \in 1 / 2 \mathbb{Z}}$ is strictly increasing and separated by $\delta^{\prime}$, and

$$
\begin{gathered}
C_{1} e^{2 \pi|y|} \leq|\Psi(x+i y)| \leq C_{2} e^{2 \pi|y|} \quad \forall x, y \in \mathbb{R}:|y| \geq 1, \\
C_{3} \leq\left|\Psi^{\prime}\left(k+\delta_{k}\right)\right| \leq C_{4} \quad \forall k \in 1 / 2 \mathbb{Z} .
\end{gathered}
$$

Proof. i) For $j \in \mathbb{Z}$ define

$$
\alpha^{j}:=\frac{N / 2-2 \sum_{k=j N+1}^{(j+1) N} \delta_{k}}{N+\delta_{(j+1) N+1}-\delta_{j N+1}} .
$$

This is well defined since $N>2 L$. Moreover

$$
\alpha^{j} \geq 1 / 4-d, \quad 1-\alpha^{j} \geq 1 / 4-d .
$$

ii) For any $k \in \mathbb{Z}$ there are unique numbers $l \in\{1, \ldots, N\}$ and $j \in \mathbb{Z}$ such that $k=j N+l$. For such $k$ define

$$
\delta_{k+1 / 2}:=-1 / 2+\left(1-\alpha^{j}\right) \delta_{k}+\alpha^{j} \delta_{k+1}+\alpha^{j} .
$$

It follows that $\left|\delta_{k+1 / 2}\right| \leq 3 / 2+2 L=L^{\prime}$, and that $\left(k+\delta_{k}\right)_{k \in 1 / 2 \mathbb{Z}}$ is a strictly increasing sequence, separated by $\delta^{\prime}$.

iii) For $j \in \mathbb{Z}$ define

$$
K^{j}:=1 / 2 \mathbb{Z} \cap[j N+1,(j+1) N+1) .
$$

An easy calculation shows

$$
\sum_{k \in K^{j}} \delta_{k}=0 \quad \forall j \in \mathbb{Z}
$$

iv) Since $\left(\delta_{k}\right)_{k \in \mathbb{Z}}$ is bounded, we have $\delta \leq 1$, hence $\delta^{\prime} \leq 1 / 4$. Choose $\beta \in\left[\delta^{\prime} / 4, \delta^{\prime} / 2\right]$ such that

$$
\left|k+\delta_{k}+\beta\right| \geq \delta^{\prime} / 8 \quad \forall k \in 1 / 2 \mathbb{Z} .
$$

Define $\lambda_{k}:=k+\beta$ for $k \in 1 / 2 \mathbb{Z}$. Then,

$$
\left|\lambda_{k}+\delta_{k}\right| \geq \delta^{\prime} / 8, \quad\left|\lambda_{k}\right| \geq \delta^{\prime} / 4 \quad \forall k \in 1 / 2 \mathbb{Z} .
$$


Define

$$
\begin{aligned}
\Psi_{1}(z) & :=\mathrm{P} . \mathrm{V} \cdot \prod_{k \in 1 / 2 \mathbb{Z}}\left(1-\frac{z}{\lambda_{k}+\delta_{k}}\right)\left(:=\lim _{R \rightarrow \infty} \prod_{k \in 1 / 2}\left(1-\frac{z}{\lambda_{k}, k \mid \leq R}\right)\right), \\
\Phi_{1}(z) & :=\mathrm{P} . \mathrm{V} \cdot \prod_{k \in 1 / 2 \mathbb{Z}}\left(1-\frac{z}{\lambda_{k}}\right) .
\end{aligned}
$$

It follows from general theorems on entire functions that both products converge uniformly on compact subsets of $\mathbb{C}$ and that $\Psi_{1}, \Phi_{1}$ are entire. Moreover, $\Psi_{1}$ is of exponential type (cf. LEVIN [8, pp. 30, 33]). The zeros of $\Psi_{1}$ are given by $\left(\lambda_{k}+\delta_{k}\right)_{k \in 1 / 2 \mathbb{Z}}$, the zeros of $\Phi_{1}$ are given by $\left(\lambda_{k}\right)_{k \in 1 / 2} \mathbb{Z}$. For $z \in \mathbb{C} \backslash\left\{\lambda_{k}: k \in 1 / 2 \mathbb{Z}\right\}$ we have

$$
\left|\frac{\Psi_{1}(z)}{\Phi_{1}(z)}\right|=\underbrace{\mathrm{P} . \mathrm{V} . \prod_{j \in \mathbb{Z}} \prod_{k \in K^{j}}\left|1+\frac{\delta_{k}}{\lambda_{k}-z}\right|} \cdot \underbrace{\left(\mathrm{P} . \mathrm{V} \cdot \prod_{j \in \mathbb{Z}} \prod_{k \in K^{j}}\left|1+\frac{\delta_{k}}{\lambda_{k}}\right|\right)^{-1}} .
$$

v) Let $\varepsilon>0, z=x+i y, x, y \in \mathbb{R}$, dist $\left(z,\left\{\lambda_{k}, \lambda_{k}+\delta_{k}: k \in 1 / 2 \mathbb{Z}\right\}\right) \geq \varepsilon$. Let $\lambda^{j, z}$ be an element of $\left\{\lambda_{k}: k \in K^{j}\right\}$, satisfying

$$
\left|\lambda^{j, z}-x\right|=\min _{k \in K^{j}}\left|\lambda_{k}-x\right|
$$

Denote by $K_{p}^{j}$ an arbitrary subset of $K^{j}$ with $p$ elements $(p \in\{2,3, \ldots, 2 N\})$. Then,

$$
\prod_{k \in K^{j}}\left(1+\frac{\delta_{k}}{\lambda_{k}-z}\right)=1+\sum_{k \in K^{j}} \frac{\delta_{k}}{\lambda_{k}-z}+\sum_{p=2}^{2 N} \sum_{K_{p}^{j} \subseteq K^{j}} \prod_{k \in K_{p}^{j}} \frac{\delta_{k}}{\lambda_{k}-z}=: 1+a_{j} .
$$

¿From (4) we have $\sum_{k \in K^{j}} \delta_{k} /\left(\lambda^{j, z}-z\right)=0$, hence

$$
a_{j}=\sum_{k \in K^{j}} \frac{\delta_{k}\left(\lambda^{j, z}-\lambda_{k}\right)}{\left(\lambda_{k}-z\right)\left(\lambda^{j, z}-z\right)}+\sum_{p=2}^{2 N} \sum_{K_{p}^{j} \subseteq K^{j}} \prod_{k \in K_{p}^{j}} \frac{\delta_{k}}{\lambda_{k}-z}
$$

This can be estimated by

$$
\left|a_{j}\right| \leq \frac{2 N \cdot L^{\prime} \cdot N}{\left|\lambda^{j, z}-z\right|^{2}}+\frac{2^{2 N} \cdot\left(L^{\prime}\right)^{2 N}}{\left|\lambda^{j, z}-z\right|^{2}} .
$$

Let $j_{0}$ be an integer such that $\left|\lambda^{j_{0}, z}-x\right|=\min \left\{\left|\lambda^{j, z}-x\right|: j \in \mathbb{Z}\right\}$. Then,

$$
\left|\lambda^{j_{0}+j, z}-z\right| \geq \varepsilon+(|j|-1) N \quad \forall j \in \mathbb{Z}
$$

Defining

$$
j_{1}:=\sqrt{4 L^{\prime}+2\left(2 L^{\prime}\right)^{2 N} / N^{2}}+1,
$$


(5) and (6) give $\sum_{|j| \geq j_{1}}\left|a_{j_{0}+j}\right| \leq\left(4 L^{\prime}+2\left(2 L^{\prime}\right)^{2 N} / N^{2}\right) \pi^{2} / 6$

and $\left|a_{j_{0}+j}\right| \leq 1 / 2 \forall|j| \geq j_{1}$, hence

$$
e^{-\left(L^{\prime}+\left(2 L^{\prime}\right)^{2 N} /\left(2 N^{2}\right)\right) \pi^{2}} \leq \prod_{|j| \geq j_{1}}\left|1+a_{j_{0}+j}\right| \leq e^{\left(L^{\prime}+\left(2 L^{\prime}\right)^{2 N} /\left(2 N^{2}\right)\right) \pi^{2}} .
$$

Using

$$
\left(1+L^{\prime} / \varepsilon\right)^{-2 N} \leq\left|1+a_{j}\right| \leq\left(1+L^{\prime} / \varepsilon\right)^{2 N} \quad \forall j \in \mathbb{Z}
$$

gives

$$
\left(1+\frac{L^{\prime}}{\varepsilon}\right)^{-2 N\left(2 j_{1}+1\right)} e^{-\left(L^{\prime}+\left(2 L^{\prime}\right)^{2 N} /\left(2 N^{2}\right)\right) \pi^{2}} \leq(\mathrm{I}) \leq\left(1+\frac{L^{\prime}}{\varepsilon}\right)^{2 N\left(2 j_{1}+1\right)} e^{\left(L^{\prime}+\left(2 L^{\prime}\right)^{2 N} /\left(2 N^{2}\right)\right) \pi^{2}} .
$$

For $z:=0, \varepsilon:=\delta^{\prime} / 8$, this yields an estimate for (II), which together with (7) results in

$$
D_{\varepsilon}^{-1} \leq\left|\frac{\Psi_{1}(z)}{\Phi_{1}(z)}\right| \leq D_{\varepsilon} \quad \forall z \in \mathbb{C}: \operatorname{dist}\left(z,\left\{\lambda_{k}, \lambda_{k}+\delta_{k}: k \in 1 / 2 \mathbb{Z}\right\}\right) \geq \varepsilon .
$$

vi) Define

$$
\Psi(z):=\sin \pi \beta \cdot \cos \pi \beta \cdot \Psi_{1}(z+\beta), \quad \Phi(z):=\sin \pi \beta \cdot \cos \pi \beta \cdot \Phi_{1}(z+\beta) .
$$

Then, $\Psi$ is an entire function of exponential type, real on the real axis, with zeros

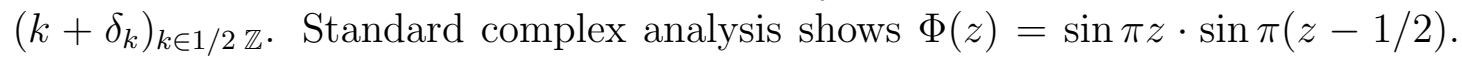
Since

$$
\left(\frac{1-e^{-2 \pi}}{2}\right)^{2} \cdot e^{2 \pi|y|} \leq|\Phi(x+i y)| \leq e^{2 \pi|y|} \quad \forall x, y \in \mathbb{R}:|y| \geq 1,
$$

(2) follows from (8) for $\varepsilon=1$.

Fix $k_{0} \in 1 / 2 \mathbb{Z}$. It is possible to choose a circle $\gamma$ with center $k_{0}+\delta_{k_{0}}$ and radius $r \in$ $\left\{\delta^{\prime} / 4, \delta^{\prime} / 2\right\}$, such that $\operatorname{dist}(\gamma, 1 / 2 \mathbb{Z}) \geq \delta^{\prime} / 8$. Then, (8) combined with estimates for $\Phi(z)$ (which follow from the canonical factorization of $\sin \pi z$ ) gives

$$
\pi^{2}\left(\delta^{\prime} / 8\right)^{2} e^{-\pi^{2} / 4} D_{\delta^{\prime} / 8}^{-1} \leq|\Psi(z)| \leq \pi^{2} / 2 \cdot e^{\pi^{2} / 4} D_{\delta^{\prime} / 8} \quad \forall z \in \gamma .
$$

If $\Psi_{k_{0}}$ is defined by

$$
\Psi_{k_{0}}(z):=\left\{\begin{array}{l}
\frac{\Psi(z)}{z-k_{0}-\delta_{k_{0}}}, z \neq k_{0}+\delta_{k_{0}} \\
\Psi^{\prime}\left(k_{0}+\delta_{k_{0}}\right), z=k_{0}+\delta_{k_{0}}
\end{array}\right.
$$

then $\Psi_{k_{0}}$ is an entire function without zeros in $\left\{z \in \mathbb{C}:\left|z-\left(k_{0}+\delta_{k_{0}}\right)\right| \leq r\right\}$. Applying the maximum/minimum principle to $\Psi_{k_{0}}$ and using (9) gives (3).

The following result represents a special case of Theorem 1.

Proposition 1. Under the assumptions of Lemma 2, $\left(e^{i\left(k+\delta_{k}\right) \bullet}\right)_{k \in \mathbb{Z}}$ is an exact frame for $L^{2}(-\pi, \pi)$ with lower frame bound

$$
A:=e^{-20 \pi^{2}\left(2 L^{\prime}\right)^{2 N} / N^{2}} \cdot\left(\delta^{\prime} /\left(9 L^{\prime}\right)\right)^{240\left(2 L^{\prime}\right)^{N}},
$$


where

$$
L^{\prime}:=3 / 2+2 L, \quad \delta^{\prime}:=(1 / 4-d) \delta
$$

Proof: The proofs of Lemmas 7 and 9 in KATSNEL'son [4] show that

$$
A_{1}:=\frac{C_{1}^{2} C_{3}^{2}}{C_{2}^{4}}\left(1+\frac{C_{4}}{C_{3}}\right)^{-1} \cdot \pi \cdot \frac{e^{-8 \pi}}{e^{8 \pi+4 \pi}-1}\left(1+\frac{2}{\delta^{\prime}}\right)^{-2}
$$

is a lower bound for $\left(e^{i\left(k+\delta_{k}\right) \bullet}\right)_{k \in \mathbb{Z}}$, where $C_{1}, C_{2}, C_{3}$ and $C_{4}$ are as in Lemma 2. Inserting the values for $C_{1}-C_{4}$ and using some suitable estimates one can show that $A_{1} \geq A$. The existence of an upper bound follows from Lemma 1. For the proof of the exactness of the frame, we refer to AvDonin [1].

Proof of Theorem 1: For $j \in \mathbb{Z}$ define

$$
S_{j}:=\{j \tilde{N}+\tilde{N}-\lceil 2 L\rceil+1, \ldots, j \tilde{N}+\tilde{N}+\lceil 2 L\rceil\} .
$$

We have $S_{j} \cap S_{j^{\prime}}=\emptyset$ for $j \neq j^{\prime}$. Define sequences $\left(\vartheta_{k}\right)_{k \in \mathbb{Z}}$ and $\left(\tilde{\delta}_{k}\right)_{k \in \mathbb{Z}}$ such that

$$
\begin{aligned}
& \vartheta_{k}:=\delta_{k} \text { if } k \in \mathbb{Z} \backslash \bigcup_{j \in \mathbb{Z}} S_{j}, \\
& \left\{\vartheta_{k}+k: k \in S_{j}\right\}=\left\{\delta_{k}+k: k \in S_{j}\right\} \forall j \in \mathbb{Z} \text { and } \vartheta_{k}+k<\vartheta_{k^{\prime}}+k^{\prime} \text { for } \\
& k, k^{\prime} \in S_{j}, k<k^{\prime}, \\
& \left\{\tilde{\delta}_{k}+k: k \in\{j \tilde{N}+1, \ldots,(j+1) \tilde{N}\}\right\}=\left\{\vartheta_{k}+k: k \in\{j \tilde{N}+1, \ldots,(j+\right. \\
& 1) \tilde{N}\}\} \forall j \in \mathbb{Z} \text { and } \\
& \tilde{\delta}_{k}+k<\tilde{\delta}_{k+1}+k+1 \forall k \in \mathbb{Z} .
\end{aligned}
$$

We obtain $\left|\tilde{\delta}_{k}\right| \leq 3 L+1 \forall k \in \mathbb{Z}$ and

$$
\left|\sum_{k=j \tilde{N}+1}^{(j+1) \tilde{N}} \tilde{\delta}_{k}\right| \leq \frac{d+1 / 4}{2} \cdot \tilde{N} \quad \forall j \in \mathbb{Z} .
$$

An application of Proposition 1 to $\left(\tilde{\delta}_{k}\right)_{k \in \mathbb{Z}}$ completes the proof of Theorem 1 .

Remark. The second part of Lemma VI in Duffin And Schaeffer [3] was proved indirectly. Since their proof rests on this lemma, they did not obtain an explicit lower bound. We will prove Theorem 2, following a construction by SEIP [9].

Proof of Theorem 2: Suppose first $\sigma>1$ and $\gamma=\pi$. Define

$$
L_{A}:=3 M-1 / 2, \quad N:=2 M(M+1), \quad d:=1 /(M+1) .
$$

Then

$$
N>2 L_{A} \cdot \frac{3 / 4+d}{1 / 4-d}
$$

and the number of points of the sequence $\left(\lambda_{n}\right)_{n \in \mathbb{Z}}$ in each interval of length $M$ is at least $M+1$. 
Following the proof of Theorem 2.3 in SEIP [9], we conclude that there is a sequence $\left(\delta_{k}\right)_{k \in \mathbb{Z}}$ of real numbers, bounded by $L_{A}$, such that $\left(k+\delta_{k}\right)_{k \in \mathbb{Z}}$ is strictly increasing, $\left\{k+\delta_{k}: k \in \mathbb{Z}\right\}$ is a subset of $\left\{\lambda_{k}: k \in \mathbb{Z}\right\}$, and

$$
\left|\sum_{k=j N+1}^{(j+1) N} \delta_{k}\right| \leq N \cdot d \quad \forall j \in \mathbb{Z}
$$

Applying Proposition 1 shows that $\left(e^{i\left(k+\delta_{k}\right)} \bullet\right)_{k \in \mathbb{Z}}$ is an exact frame for $L^{2}(-\pi, \pi)$ with lower bound $A \geq A_{D S}(L, \delta, \sigma, \pi)$. Then, $A_{D S}(L, \delta, \sigma, \pi)$ is a lower bound for $\left(e^{i \lambda_{n} \bullet}\right)_{n \in \mathbb{Z}}$ as well. The existence of an upper bound follows from Lemma 1. This proves Theorem 2 for $\sigma>1, \gamma=\pi$. The general case follows by a dilation.

Remark. SEIP [9, Theorem 2.3] proved that, under the conditions of the theorem of Duffin and Schaeffer, there is a subsequence $\left(\lambda_{n_{k}}\right)_{k \in \mathbb{Z}}$, such that $\left(e^{i \lambda_{n_{k}} \bullet}\right)_{k \in \mathbb{Z}}$ is an exact frame for $L^{2}(-\gamma, \gamma)$. The proof of Theorem 2 even shows that $A_{D S}(L, \delta, \sigma, \gamma)$ is a lower bound for this exact frame.

Open question. Can sharp frame bounds be obtained, for the Theorems of Avdonin and of Duffin and Schaeffer? (The lower bounds, obtained in Theorems 1 and 2, are not sharp.)

\section{An application to irregular sampling}

Suppose $\left(\lambda_{n}\right)_{n \in \mathbb{Z}}$ is a sequence of real numbers such that $\left(e^{i \lambda_{n} \bullet}\right)_{n \in \mathbb{Z}}$ is an $(A, B)$ frame for $L^{2}(-\sigma, \sigma)$. Define $\varphi_{n}$ by

$$
\varphi_{n}(z):=\left\{\begin{array}{l}
\frac{\sigma}{\pi} \cdot \frac{\sin \sigma\left(z-\lambda_{n}\right)}{\sigma\left(z-\lambda_{n}\right)}, z \neq \lambda_{n} \\
\frac{\sigma}{\pi}, z=\lambda_{n}
\end{array}\right.
$$

Then, $\varphi_{n} \in P W_{\sigma}^{2}$ and $\left(\varphi_{n}\right)_{n \in \mathbb{Z}}$ is an $\left(\frac{A}{2 \pi}, \frac{B}{2 \pi}\right)$-frame for $P W_{\sigma}^{2}$.

If $S: P W_{\sigma}^{2} \rightarrow P W_{\sigma}^{2}, f \mapsto \sum_{n \in \mathbb{Z}} f\left(\lambda_{n}\right) \varphi_{n}$, is the frame operator, corresponding to this frame, then $S$ is a bijective bounded linear operator satisfying $\left\|S^{-1}\right\| \leq 2 \pi / A$ (cf. Benedetto and Walnut [2, Theorem 3.2], Duffin and Schaeffer [3, Section 3], KöLzOW [5, Section II.1]). Define

$$
s_{n}(f):=\sum_{|k| \leq n} f\left(\lambda_{k}\right) S^{-1} \varphi_{k}, \quad f \in P W_{\sigma}^{2}, \quad n \in \mathbb{N} .
$$

Then, $\left(s_{n}(f)\right)_{n \in \mathbb{N}}$ converges to $f$ in the $P W_{\sigma}^{2}$-norm. For every $n \in \mathbb{N}, \| s_{n}(f)-$ $f \|_{P W_{\sigma}^{2}}$ is called the $n$th truncation error.

Proposition 2. Suppose $\left(\lambda_{n}\right)_{n \in \mathbb{Z}}$ is a sequence of real numbers such that $\left(e^{i \lambda_{n} \bullet}\right)_{n \in \mathbb{Z}}$ is an $(A, B)$-frame for $L^{2}(-\sigma, \sigma)$. Then, the $n$th truncation error satisfies

$$
\left\|s_{n}(f)-f\right\|_{P W_{\sigma}^{2}} \leq \sqrt{\frac{2 \pi}{A}} \cdot\left(\sum_{|k|>n}\left|f\left(\lambda_{k}\right)\right|^{2}\right)^{1 / 2} \quad \forall f \in P W_{\sigma}^{2} .
$$


The first version of Proposition 2, proposed by BITTNER (private communication), contained the factor $\sqrt{2 \pi B} / A$, instead of $\sqrt{2 \pi / A}$. The latter was suggested by the referee, refering to the fact that $T: P W_{\sigma}^{2} \rightarrow l^{2}(\mathbb{Z}), f \mapsto\left(f\left(\lambda_{n}\right)\right)_{n \in \mathbb{Z}}$ is a bounded injective linear operator with bounded inverse on its range satisfying $\left\|T^{-1}\right\| \leq \sqrt{2 \pi / A}$.

Acknowledgments. The author would like to thank D. Kölzow for his steady support, and B. Bittner as well as the referee for their suggestions.

\section{References}

[1] S. A. Avdonin. 1974. "On the question of Riesz bases of exponential functions in $L^{2} . "$ Vestnik Leningrad Univ. Ser. Mat., 13, pp. 5 - 12 (Russian); English translation in Vestnik Leningrad Univ. Math., 7 (1979), pp. 203 211.

[2] J. J. Benedetto, D. F. Walnut. 1994. "Gabor frames for $L^{2}$ and related spaces", in Wavelets: Mathematics and Applications, J. J. Benedetto, M. W. Frazier, eds., CRC Press, Boca Raton, pp. 97 - 162.

[3] R. J. Duffin, A. C. Schaeffer. 1952. "A class of nonharmonic Fourier series." Trans. Amer. Math. Soc., 72, pp. $341-366$.

[4] V. É. Katsnel'son. 1971. "Exponential bases in L2." Funkcional'. Analiz Prilozenija, 5, No. 1, pp. 37 - 47 (Russian); English translation in Functional Analysis Appl., 5 (1971), pp. 31 - 38.

[5] D. KöLzow. 1994. Wavelets. A tutorial and a bibliography. Rend. Ist. Matem. Trieste, 26, Suppl.

[6] D. KöLzow. 1996. Analysis-Seminar über Abtastsätze, Wintersemester 1996/ 97, Univ. Erlangen-Nürnberg.

[7] B. Ya. Levin. 1961. "On bases of exponential functions in $L^{2}$." Zap. Har'kov. Gos. Univ. i Har'kov. Mat. Ob̌̌č. (4), 27, pp. 39 - 48. (Russian)

[8] B. Ya. Levin. 1996. Lectures on entire functions. Translations of Mathematical Monographs, 150, American Mathematical Society.

[9] K. SEIP. 1995. "On the connection between exponential bases and certain related sequences in $L^{2}(-\pi, \pi)$." J. Funct. Anal., 130 , pp. 131 - 160.

[10] R. M. Young. 1980. An introduction to nonharmonic Fourier series. Academic Press, New York.

Alexander M. Lindner

Mathematisches Institut, Universität Erlangen-Nürnberg

Bismarckstraße 1 1/2

D-91054 Erlangen GERMANY

e-mail: lindnera@mi.uni-erlangen.de 